Running head: THE SPATIAL SPREAD OF ATTENTION AND AGEING

\title{
Changes in the spatial spread of attention with ageing
}

Rebecca K. Lawrence, Mark Edwards, and Stephanie C. Goodhew

Research School of Psychology, The Australian National University

Corresponding Author: Rebecca K. Lawrence

Address: Research School of Psychology (Building 39)

The Australian National University, Canberra, 2601

Phone: +6102 61254582

Email:Rebecca.Lawrence@anu.edu.au 
THE SPATIAL SPREAD OF ATTENTION AND AGEING

\begin{abstract}
Spatial attention is a necessary cognitive process, allowing for the direction of limited capacity resources to varying locations in the visual field for improved visual processing. Thus, understanding how ageing influences these processes is vital. The current study explored the relationship between the spatial spread of attention and healthy ageing using an inhibition of return task to tap visual attention processing. This task allowed us to measure the spatial distribution of inhibition, and thus acted as a marker for attentional spread. Past research has indicated minimal age differences in inhibitory spread. However, these studies used placeholder stimuli, which may have restricted the range over which age differences could be reliably measured. To address this, in Experiment One, we measured the relationship between the spatial spread of inhibition and healthy aging using a method which did not employ placeholders. In contrast to past research, an age difference in inhibitory spread was observed, where in comparison to younger adults, older adults exhibited a relatively restricted spread of attention. Experiment Two then confirmed these findings, by directly comparing inhibitory spread for placeholder present and placeholder absent conditions, across younger and older adults. Again, it was found that age differences in inhibitory spread emerged, but only in the placeholder absent condition. Possible reasons for the observed age differences in attention are discussed.
\end{abstract}

Keywords: spatial attention, aging, attention distribution 
THE SPATIAL SPREAD OF ATTENTION AND AGEING

Selective spatial attention allows for the allocation of the brain's finite cognitive resources for efficient processing of relevant visual information, while filtering out irrelevant visual noise (Broadbent, 1982; Carrasco, 2011; Desimone \& Duncan, 1995; Kastner \& Pinsk, 2004; Posner, 1980; Posner, Snyder, \& Davidson, 1980). The aim of the current study was to clarify the relationship between healthy ageing and the dynamics of one aspect of selective spatial attention: the distribution of attention across space. This is important because the relative distribution of spatial attention may underscore differences in visual search efficiency, perceptual sensitivity, distractor processing, and working- memory capacity (e.g. Bleckley, Durso, Crutchfield, Engle, \& Khanna, 2003; Cave \& Chen, 2016; Eriksen \& James, 1986; Goodhew, Lawrence, \& Edwards, 2017; Goodhew, Shen, \& Edwards, 2016; Greenwood \& Parasuraman, 1999, 2004; Hoyer, Cerella, \& Buchler, 2011; Pringle, Irwin, Kramer, \& Atchley, 2001; Theeuwes, Kramer, \& Belopolsky, 2004). It is therefore imperative that the operation of attentional spread with ageing is understood in great detail. Yet current evidence for changes in attentional distribution across the lifespan is mixed. While some studies reveal substantial age differences in the capacity to spread spatial attention (Gottlob \& Madden, 1999; Greenwood \& Parasuraman, 1999, 2004; Hüttermann, Bock, \& Memmert, 2012; Kosslyn, Brown, \& Dror, 1999; Pesce, Guidetti, Baldari, Tessitore, \& Capranica, 2005), others indicate none, or only small differences (Hartley, Kieley, \& Mckenzie, 1992; Hartley \& Kieley, 1995; Langley, Gayzur, Saville, Morlock, \& Bagne, 2011; Madden \& Gottlob, 1997; McCalley, Bouwhuis, \& Juola, 1995; Quigley, Andersen, \& Müller, 2012).

Previous research has demonstrated a strong link between working memory capacity and visual attention (Bleckley et al. 2003; Kreitz, Furley, Memmert, \& Simons, 2015). Likewise, there are well known declines in working memory capacity with age (Hedden \& 
THE SPATIAL SPREAD OF ATTENTION AND AGEING

Gabrieli, 2004; Mattay et al., 2006; Verhaeghen \& Salthouse, 1997). Therefore, the contradictory effects of ageing on attentional spread are surprising. That is, if changes in working memory capacity do underlie changes in attentional spread, one would expect more consistent age differences in the literature, with older adults showing differences in the distribution of attention across space (Rolle, Anguera, Skinner, Voytek, \& Gazzaley, 2017). Here, similar to Rolle et al. (2017), and Erel and Levy (2016), we believe that one reason for these contradictory findings are the different methods which have previously been used to claim age equivalency in the spatial distribution of attention. Specifically, we believe that these methods may not have been sensitive enough to uncover the potentially subtle, and fine-grained changes in attentional processing across the lifespan.

A key method utilised to explore the dynamics of visual attention is the spatial-cueing paradigm (Posner, 1980; Posner \& Cohen, 1984). Here, attention is oriented via a noninformative peripheral cue to a potential target location. Following this, a target is presented at either the same (valid) or different (invalid) location to the cue. Target detection response times are then compared between validly and invalidly cued locations. When the target is presented shortly after the cue, a facilitation effect is observed, where response times are faster for the validly cued compared to invalidly cued location. However, if the target is presented approximately 300ms or more following the cue, an inhibitory effect is observed, where target detection response times are comparatively slower. This is labelled Inhibition of Return (IOR), and is theorised to reflect an effective reorienting of visual attention to novel spatial locations (Berlucchi, 2006; Posner, Rafal, Choate, \& Vaughan, 1985; Klein \& MacInnes, 1999).

The spatial cueing paradigm can be used to quantify attentional spread by measuring the relative change in either facilitation or inhibition surrounding an attended location (e.g. 
THE SPATIAL SPREAD OF ATTENTION AND AGEING

Bennett \& Pratt, 2001; Downing, 1988; Klein, Christie, \& Morris, 2005; LaBerge, 1983; LaBerge \& Brown, 1989). That is, while the strength of attention is strongest at the location of the cue, attentional effects also spread to regions surrounding the cue. This is seen via a change in response time as the spatial disparity between the cued and the target location grows. The gradient change in attention across space can be used to infer attentional spread, and to compare both individual and group differences in spatial attention (for example, see Bennett \& Pratt, 2001; Taylor, Chan, Bennett, \& Pratt, 2015; Wilson, Lowe, Ruppel, Pratt, \& Ferber, 2016). Regression coefficients describing the slope between cue-target distance and reaction time are calculated for population groups. These coefficients are then compared between groups to infer potential differences in the 'roll-off' of attentional resources. A higher value coefficient indicates a steeper rate in the decline of visual attention across space, and therefore, a relatively restricted spread of attention, while a lower value coefficient suggests a comparatively shallower drop off in attention, implying a broader spread of visual attention resources (Wilson et al., 2016).

Although we acknowledge that there is an oculomotor component to IOR, here, we are primarily interested in the attentional component of IOR (e.g. Hunt \& Kingstone, 2003; Kingstone \& Pratt, 1999). That is, we wish to explore location based IOR patterns when eye movements are restricted. This will allow us to measure a form of IOR more closely related to covert orienting (e.g., see Chica, Taylor, \& Klein, 2010). Similar work has recently been conducted by Wilson and colleagues (2016). Here, inhibitory slopes were used to explore potential personality differences in the distribution of attention. They measured the big five personality traits (Neuroticism, Extraversion, Openness, Conscientiousness, and Agreeableness; Digman, 1990), and then correlated these with individual IOR gradients. Importantly, by measuring a form of IOR which emphasised covert attention, the authors 
THE SPATIAL SPREAD OF ATTENTION AND AGEING

were able to equate their results to attentional spread. That is, the slope of IOR was seen as inferring previous preferential attending, and thus, indicative of how attention may have been initially distributed when initially oriented to a cued location. Overall, Wilson and colleagues found that personality traits Openness and Conscientiousness predict IOR slope, where those with higher Openness scores had broader attention, and those with higher conscientiousness scores had narrower attention. This demonstrates how the spatial distribution of IOR can be used to draw inferences about individual and group differences in the spatial spread of attention.

Studies comparing differences in younger and older adult's spread of attention have predominantly found only minimal changes in the gradient of both facilitation and inhibition, regardless of the manipulation of attentional spread, and have concluded that the distribution of attention surrounding a cued region is equivalent with ageing (Hartley et al., 1992; Hartley \& Kieley, 1995; Langley et al., 2011). Here, we will focus on analysing previous work which measures age changes in the spread of IOR, as this is the primary measure of interest used in the current study. We have chosen to measure IOR, instead of facilitation, as the longer time course of IOR means that attention can be sufficiently spread around the cued location to measure age differences with a high level of sensitivity (Wilson et al., 2016). Likewise, as Jefferies, Roggeveen, Enns, Bennett, Sekuler, and Di Lollo (2015) have shown that older adults take slightly longer to contract attention, the longer time course of the IOR paradigm seems most appropriate.

To our knowledge, only two studies to date have directly examined the effect of aging on the spatial distribution of IOR (Hartley \& Kieley, 1995; Langley et al., 2011). In both studies, while aging influenced response times, such that older adults had slower target detection speeds than younger adults, it was concluded that aging did not influence the 
THE SPATIAL SPREAD OF ATTENTION AND AGEING

distribution of IOR. This conclusion was reached, even though some of the experiments within these studies did in fact, find an age change in response time, as the distance between the cue and target grew (although this finding was not consistent). Given that the spatial spread of IOR can be used to infer the spread of visual attention across space, these mixed findings indicate that the spatial distribution of attention did not reliably vary as a function of age. However, both studies employed a particular methodology, which recent evidence suggests may constrain the spread of attention across space. That is, in both Hartley and Kieley (1995), and Langley et al. (2011), attention was cued via the brightening placeholder boxes (see Figure 1). Research examining the influence of placeholders on the spatial distribution of inhibition in a younger adult sample suggests that placeholders may limit the bounds that attentional resources spread to in the visual field (Taylor et al., 2015). In other words, the presence versus absence of placeholders can result in qualitatively different effects of visual attention (Hilchey, Pratt, \& Christie, 2016; Nicol, Watter, Gray, \& Shore, 2009; Taylor et al., 2015). For instance, although not directly comparing attentional slopes, Hilchey et al. (2016) found that the magnitude of IOR is often greater when placeholders are present in the display. Thus, the conclusions of both Langley et al (2011), and Hartley and Kieley (1995) may not be generalizable to placeholder absent conditions.
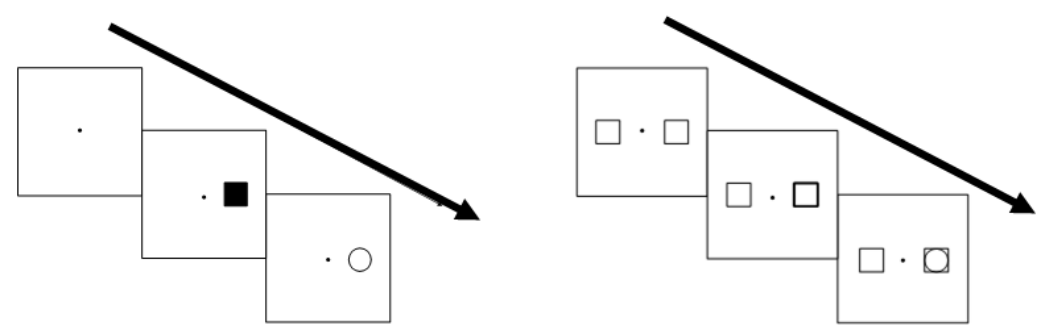
THE SPATIAL SPREAD OF ATTENTION AND AGEING

Figure 1. Placeholder absent (left) and Placeholder present (right) cueing paradigms. In the placeholder absent condition, a transient stimulus, the square, is presented to attract attention. In the placeholder present condition, attention is attracted through the brief brightening of a placeholder box, indicated by the bolded line. In both conditions, the participant's task is to detect the circle as quickly as possible.

More specifically, in Taylor et al. (2015), attention was cued to one of four possible locations, with target detection measured at 121 possible locations. In the placeholder present condition, possible cued locations were drawn and remained on the visual display for the duration of the trial. In the placeholder absent condition, the cue location was presented briefly, and did not remain in the visual display. Crucially, in the placeholder present condition, response times to target detection at placeholder locations were significantly slower compared to target detection outside of placeholder locations, irrespective of the placeholder cued. However, in the placeholder absent condition, response times to target detection decreased as cue-target distance increased, regardless of whether the target fell in one of the four potential cue locations. Thus, placeholder presence markedly influenced the distribution of IOR, such that attention was affixed to the location of the placeholders, lowering the sensitivity of the model to describe the cue-target distance relationship. This suggests that when measuring the gradient of spatial inhibition, the presence of placeholders may potentially conceal fine-grained changes in inhibitory spread at increasing cue-target distances, by anchoring attention to the bounds of placeholders. In turn, this may lead to inconsistent findings in the influence of attentional cueing on the distribution of inhibition.

Likewise, research emphasising the role of placeholders in inducing regions of attentional facilitation strongly indicate that the spatial structure imposed by placeholders could limit the spatial spread of attention (Egly \& Homa, 1984; Jefferies \& Di Lollo, 2015; 
THE SPATIAL SPREAD OF ATTENTION AND AGEING

McCalley et al., 1995). For example, studies conducted by Jefferies and colleagues found that observers can adopt an annular distribution of attention, inhibiting a central stream of letters, only when placeholders were continuously displayed in the visual scene. Without these placeholders, observers were relatively more distracted by the central visual stimuli (Jefferies \& Di Lollo, 2015; Jefferies \& Di Lollo, 2017). Thus, placeholder arrays can be utilised by participants to accurately deploy attentional resources to regions most conducive to accurate task performance, thus limiting the bounds of attentional spread. Work conducted by McAuliffe, Chasteen, and Pratt (2006) further shows that the use of placeholders to cue attention in younger and older adults has significant effects on IOR patterns, compared to when no placeholders are present (although note, the spatial distribution of inhibition was not manipulated here). Here, IOR magnitudes for placeholders and no placeholders were compared between younger and older adults. Younger adults showed stronger IOR magnitudes for placeholder present conditions, while older adults showed no difference. Taken together, it is clear that to fully understand age changes in the spread of attention, these phenomena must be compared for placeholder absent conditions, which more closely measure location based IOR.

Therefore, the focus of the present study was to compare the spatial distribution of IOR when placeholders were absent from the visual display, for younger versus older adults. This experimental method is largely identical to that used by Wilson and colleagues to measure personality differences in the spatial spread of attention (Wilson et al., 2016). Here, however, age differences, rather than individual differences in personality, were the comparison of interest on this metric. Experiment 1 compared inhibitory spread for younger and older adults when placeholders were absent from the display. Experiment 2 then directly 
THE SPATIAL SPREAD OF ATTENTION AND AGEING

compared older and younger adults inhibitory spread for both placeholder-present, and placeholder-absent conditions.

\section{Experiment 1}

Here, we aimed to compare inhibitory spread for older and younger adults, when placeholders were absent from the visual display. As shown in Figure 2, on each trial, spatial attention was cued to one of four locations via the brief presentation of a small green circle. Following a delay of $1315 \mathrm{~ms}$, the target, a small white circle, was presented at one of 40 possible locations. This allowed for a large range of cue-target distances to be sampled, giving a highly sensitive measure of attentional spread. Furthermore, in contrast to previous studies examining age differences in the spatial spread of IOR, eye movements were recorded, and we encouraged participants to minimise eye movements during practice trials, as well as throughout the study, to be more confident that covert attention was measured. As emphasised earlier, the primary reason we have chosen to do this is because we wanted to ensure that the process we are measuring closely resembles covert attentional processes, which may be likened to the spatial distribution of attentional facilitation (although see Hilchey et al., 2014 for an alternate explanation). Finally, to ensure that age differences in vision did not unduly influence our findings (see Owsley, 2011 for a review), stimulus saliency was emphasised, using brightly coloured stimuli on black backgrounds (i.e., high contrast), with stimuli presented largely within para-foveal rather than peripheral vision.

To compare attentional gradients, regression coefficients describing the relationship between distance and response time will be compared between age groups. If age does not 
THE SPATIAL SPREAD OF ATTENTION AND AGEING

influence the spread of IOR, we expect no age differences in coefficients modelling distanceresponse time, regardless of the absence of placeholders. This would provide further support to claims that the spatial spread IOR remains stable with healthy aging. If, however, age differences in the spatial spread of inhibition exist, we expect these coefficients to differ significantly. If older adults have a relatively larger coefficient, this would suggest that they have a more focal distribution of inhibition. In contrast, if older adults have a relatively smaller coefficient, this would suggest they adopt a more diffuse spread of inhibition. In turn, such a finding would support the broader literature claiming age differences in attentional distribution, which may potentially be linked to differences in working memory capacity.

\section{Method}

\section{Participants}

Eighty-three participants from the Australian National University and the general public participated in the study, and provided written informed consent. The ethical aspects of the research were approved by the Australian National University Higher Research Ethics Committee. Persons aged 18 to 30 years were recruited for the younger adult sample, and persons aged 60 years and over were recruited for the older adult sample. The younger adult group comprised of 42 participants, and the older adult group comprised of 41 participants. Participants completed a series of questionnaires designed to capture personality, health, and cognitive traits. These were the 15 item geriatric depression scale (GDS; Yesavage \& Sheikh, 1986), the Mini Mental State Examination (MMSE; Folstein, Folstein, \& McHugh, 1975), and demographic and health questionnaire designed by the research team. This questionnaire asked participants to indicate the presence of any physical (including eye health), cognitive, 
THE SPATIAL SPREAD OF ATTENTION AND AGEING

or psychological illnesses by ticking the appropriate box. A summary of demographic data for the participant groups used in the main analysis is presented in Table 1.

Table 1

Demographic data of participants included in final analyses of Experiment 1

$\mathrm{M}=20.16(\mathrm{SD}=1.77)$

Education

Ethnicity

Gender

GDS

MMSE

Eye Health

Hearing Impairment

Cognitive Health

Psychological

Illness

High

Blood
Younger

$\mathrm{M}=14.84(\mathrm{SD}=1.93)$

$\mathrm{M}=17.41(\mathrm{SD}=6.53)$

.040

Older

$M=70.88(S D=6.25)$

$$
\text { M }=17.41(\mathrm{SD}=6.53)
$$

(2)

$<.001 *$

Female $=20$

Female $=22$

.492

$$
\mathrm{M}=4.36(\mathrm{SD}=3.40) \quad \mathrm{M}=.75(\mathrm{SD}=1.16) \quad<.001 *
$$

$\mathrm{M}=29.55(\mathrm{SD}=0.83)$

$\mathrm{M}=29.44(\mathrm{SD}=0.95)$

.627

sig.

$$
\mathrm{N}=0
$$

$$
\mathrm{N}=5
$$

$$
\mathrm{N}=0
$$$$
\mathrm{N}=8
$$

$\mathrm{N}=0$

$$
\mathrm{N}=1
$$

$$
\mathrm{N}=4
$$

$$
\mathrm{N}=3
$$


THE SPATIAL SPREAD OF ATTENTION AND AGEING

Pressure

Diabetes

$\mathrm{N}=2$

$\mathrm{N}=1$

Arthritis

$\mathrm{N}=0$

$\mathrm{N}=19$

Notes. Ethnicity refers to whether participants identified with an Eastern or Western culture, or both. Eye health refers to participants who reported having cataracts, glaucoma or macular degeneration. Cognitive health refers to participants who reported having Parkinson's disease, dementia, or mild cognitive impairment. To test between group differences, independent t-tests were conducted for continuous variables. Where necessary, these tests were corrected for violations of equal variance. Two participants did not report their specific age, or total years of education. One participant did not report ethnicity.

\section{Stimuli and Apparatus}

Stimuli were presented on a LCD monitor, with a refresh rate of $59 \mathrm{~Hz}$ set to a black background, with a luminance $0.94 \mathrm{~cd} / \mathrm{m}^{2}$. The physical dimensions of the monitor were $520 \mathrm{~mm}$ by $325 \mathrm{~mm}$. To ensure that participants centred their gaze for the duration of each trial, eye movements were recorded using an Eyelink 1000 eye tracker (SR-Research, Kanata, Canada). Participants were seated comfortably for the duration of the experiment, and the viewing distance of the stimuli was fixed using a forehead and chin rest situated 940mm from the monitor. This ensured that all participants' eyes were fixated on the centre of the monitor, and that eye position was consistent across participants relative to the eye tracker. Participants wore glasses if necessary. 
THE SPATIAL SPREAD OF ATTENTION AND AGEING

MATLAB, the Psychophysics Toolbox (Brainard, 1997), and the Eyelink Toolbox (Cornelissen, Peters, \& Palmer, 2002) were used to generate the stimuli and record eye movements. To determine the location of presented stimuli, an invisible $6 \times 6$ grid, subtending $9.65^{\circ} \times 9.65^{\circ}$ of visual angle was drawn and centred on fixation. As shown in Figure 3, the cue, an unfilled green circle, with a luminance of $189.28 \mathrm{~cd} / \mathrm{m}^{2}$, and a diameter subtending approximately $0.5^{\circ}$ of visual angle, was presented at one of four possible locations, positioned at the centre of each quadrant of the grid. The target, a filled white circle with a luminance of $273.86 \mathrm{~cd} / \mathrm{m}^{2}$, and diameter subtending $0.5^{\circ}$ of visual angle, could appear at either the centre of each quadrant, or at one of the 36 locations where the invisible grid lines intersected. On each trial, the assignment of the cue and target locations was pseudorandom, with the constraint that the cue fell at each of the four potential cued locations equally as often, the cue and the target fell at the same location on $12.5 \%$ of trials, and the cue and target appeared at differing locations on remaining trials $87.5 \%$ of trials. This meant that on average, on $3.15 \%$ trials, the target fell at the same location as the cue, and that the target fell at each one of the 39 target locations on $2.24 \%$ of trials. Therefore, even though it was more likely that a target would appear at a location other than the cue, given the large number of potential non-cue locations, on each trial, it was more likely that the target appeared at the cued location rather than any other location. 


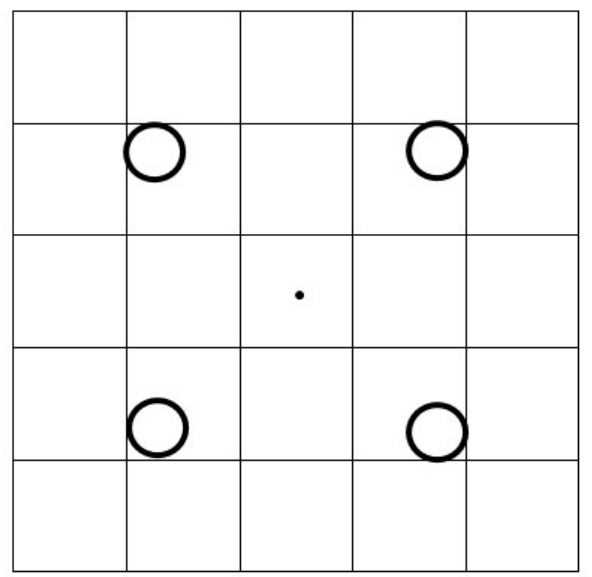

Figure 2. Potential target locations used in the current study. Targets could appear at any locations in the visual field where grid lines intersected, or at one of the four cue locations (represented by the unfilled circles, enlarged for illustrative purposes). This lead to 40 possible target locations, and 21 possible cue-target distances.

\section{Procedure}

The experiment consisted of three blocks: a calibration block, a practice block, and an experimental block. For the calibration block, a standardized 9-point calibration was completed to confirm that the eye tracker was accurately recording the participant's visual gaze. Calibration was repeated until this requirement was met. However, for 14 participants (7 older, 7 younger), after repeated attempts at calibration, eye movements were unable to be accurately tracked. These participants were given the option of completing the study without their eye movements being recorded. As the current study is interested in covert attention, and controlling for eye movements, their data are not reported here. This leaves a total sample of 69 participants (34 older, 35 younger). 
THE SPATIAL SPREAD OF ATTENTION AND AGEING

Following the calibration block, participants completed a practice block of the IOR task, consisting of approximately 10 to 20 trials. Of the practice (and subsequent experimental) trials, $80 \%$ included both the cue and the target trials, and $20 \%$ contained only the cue (catch trials). The trial sequence as shown in Figure 2 was as follows: Firstly, for the trial to begin, participants were required to focus their gaze on a ring presented at the centre of the screen and press space bar. Participants gaze was required to be within a circular region with a 2 degree radius for a trial to begin. Only when the eye-tracker registered this did a trial begin. Once the trial began, the central fixation ring changed to a fixation point which was shown for $1000 \mathrm{~ms}$. The cue was then displayed for $100 \mathrm{~ms}$. Following this, on target-present trials, a cue-to-target delay of $1315 \mathrm{~ms}$ was used to produce the IOR effect. Although it is important to recognise that the gradient of IOR differs with different cue-target delays (e.g. Taylor et al., 2015), here, we chose a single time delay designed to maximise our chance of reliably measuring age differences in attentional distribution. That is, the delay time was chosen based on earlier research indicating that maximal IOR effects with minimal differences in IOR magnitude across the two age groups would be present with this time delay (Castel, Chasteen, Scialfa, \& Pratt, 2003). Finally, on target present trials, the target was presented and participants were required to press the space bar on a keyboard as soon as they had detected the target. The target was presented until the participant responded, or 1000 ms elapsed. For cue absent trials, the 1315 ms delay was presented, as well as a blank screen for $1000 \mathrm{~ms}$. In the target absent trials, participants were asked to not respond. In the practice block, corrective feedback was given based on the participant's response. Together, the calibration and practice block typically took between 10 to 20 minutes.

Once participants understood the task, they completed the experimental block, which consisted of 410 trials. The parameters of the experimental block were identical to the 
THE SPATIAL SPREAD OF ATTENTION AND AGEING

practice block, except that no corrective feedback was given. The independent variables were cue and target location, which determined the spatial distance between cue and target, and the dependent variables were accuracy for target detection, and response time. Self-paced rest breaks were offered to the participant every $10 \%$ of trials completed. Further to this, where necessary, eye movements were recalibrated with the eye tracker throughout the study. Overall the experiment took about 1.5 hours to complete.

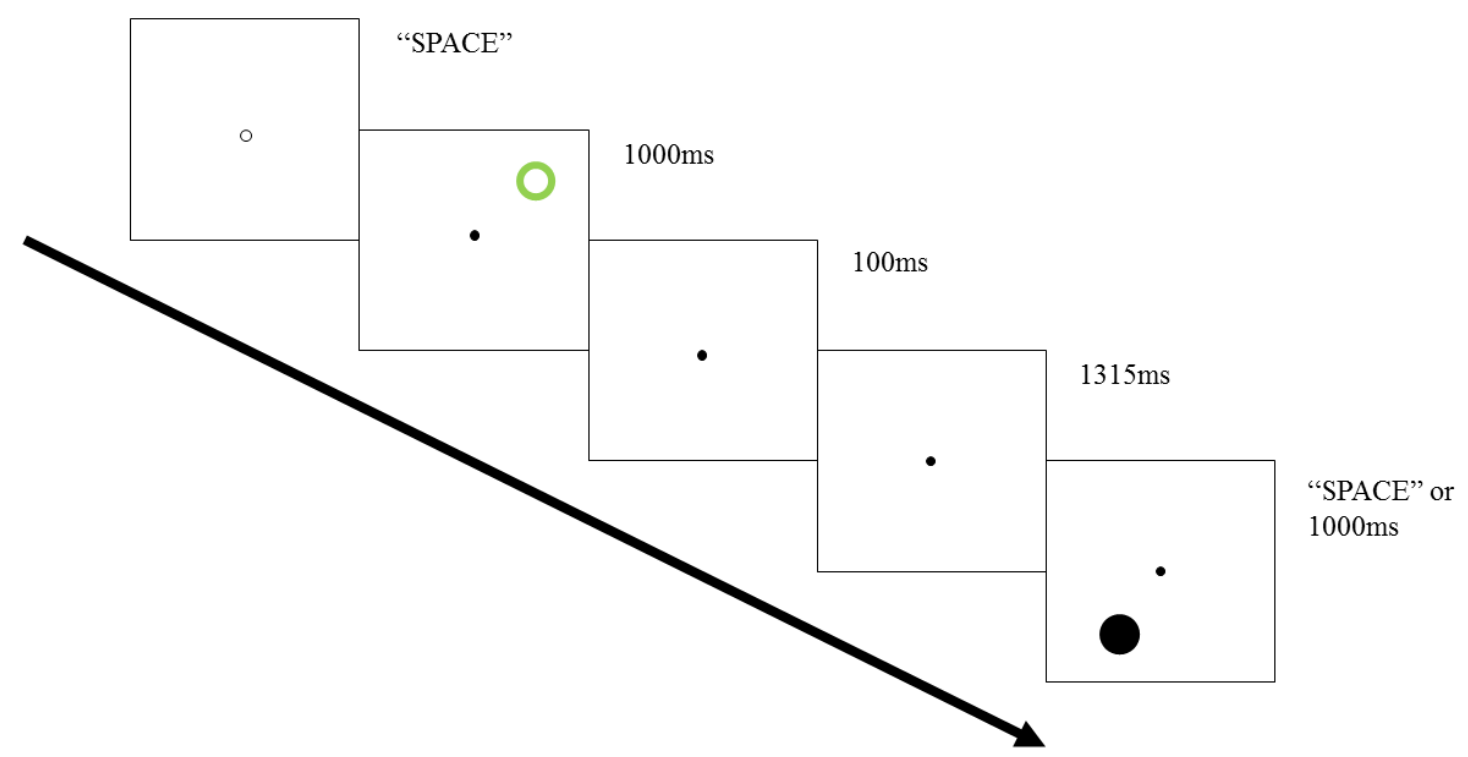

Figure 3. The IOR method used in the current study. At the beginning of each trial, participants complete a drift correction with the eye tracker to ensure accurate recordings of eye movements. After this, participants pressed the spacebar on the keyboard to begin a trial. For the duration of the trial, participants are required to maintain fixation. A transient exogenous cue, the green circle, is briefly flashed at one of four possible locations, followed by a blank interval of approximately 1315ms. Following this, the target, a white circle, is 
THE SPATIAL SPREAD OF ATTENTION AND AGEING

presented at one of 40 possible locations, until the participants detects it, via the pressing of the spacebar, or $1000 \mathrm{~ms}$ has passed.

\section{Results and Discussion}

\section{Eye Movement Data}

To measure covert attention, for each participant, a trial level analysis of eye movement data was conducted. For each trial, we determined whether a participant maintained fixation on the centre of the screen, or shifted their eye movements away from this location. If participants fixated for more than approximately $75 \mathrm{~ms}$ outside of a square $2.07^{\circ 2}$ interest area (IA), drawn around the central fixation point, eye movement data for the trial was scored as a 0 . If the participant's gaze remained in the IA, eye movement data for the trial was scored as a 1 . To check that we were not inaccurately scoring trials as zero due to eye blinks, or glare from glasses, the fixation time of approximately $75 \mathrm{~ms}$ outside of the IA was chosen.

It is important to note that we did not recycle trials where participants moved from the IA as we believed that this would be too demanding and risk undue fatigue in participants. That is, since it took approximately one hour and 15 minutes to complete the computer task, we deemed it inappropriate to continue to require participants (especially older participants) to persevere with the task longer than necessary. Likewise, in order to retain the largest amount of data as possible, rather than removing trials where participants failed to maintain fixation in the central IA, instead, we statistically controlled for any eye movements made in our main analysis. This is because using a trial removal approach may have not left enough data to reliably measure IOR effects and attentional spread at the individual level. 
THE SPATIAL SPREAD OF ATTENTION AND AGEING

\section{Accuracy Data}

Next, accuracy data was analysed. Firstly, participants were removed from further analysis if their accuracy in target-present trials fell below $90 \%$ (i.e., if they failed to respond on more than $10 \%$ of the target-present trial). This was done to maximise the possibility that all participants included in later analysis were actively engaged in the task. One older participant was removed from the data set. Likewise, in the target-absent condition, participants' data were removed from further analysis if their accuracy fell below 90\% (in other words, if they failed to withhold responses on more than $10 \%$ of the target-absent ‘catch' trials). This allowed us to control for anticipatory strategies participants may have adopted while completing the IOR task. A further three participants were removed from the data set for this reason (2 younger, 1 older), leaving a data set of 65 participants (32 older, 33 younger). For the remaining data, an independent $t$-test, with unequal variances corrected for, indicated a marginally significant difference in accuracy between the older and younger adult groups, $t(54.90)=-1.88, p=.066, d=0.47$ where older adults were slightly less accurate $\left(M_{\text {older }}=98.74 \%, S D_{\text {older }}=1.57 \%, M_{\text {younger }}=99.37 \%, S D_{\text {younger }}=1.08 \%\right)$. Likewise, an independent samples $t$-test on 'catch' trial accuracy indicated no significant difference in performance between age groups for this task, $t(63)=-0.823, \mathrm{p}=.413, d=0.21, \quad\left(M_{\text {errorolder }}=\right.$ $\left.1.75 \%, S D_{\text {errorolder }}=1.91 \%, M_{\text {erroryounger }}=2.18 \%, S D_{\text {erroryounger }}=2.26 \%\right)$.

\section{Response Time Data}

Following this, response time data was analysed to confirm an overall IOR effect for participants, and to explore whether any potential age differences existed in the magnitude of this IOR effect. Z-scores were calculated for each participant's response times across all cuetarget distances. Any Z-scores exceeding +/- 3.29 were excluded from further analysis. For the remaining data sets, overall mean reaction times were calculated. An independent samples 
THE SPATIAL SPREAD OF ATTENTION AND AGEING

$t$-test indicated a difference between older and younger adults, $t(63)=4.61, p<.001, d=$ 1.14 , where older adults were slower than younger adults, $\left(M_{\text {older }}=484 \mathrm{~ms}, S D_{\text {older }}=78 \mathrm{~ms}\right.$, $\left.M_{\text {younger }} 398 \mathrm{~ms}, S D_{\text {younger }}=72 \mathrm{~ms}\right)$. Next, mean response times for cued versus uncued locations were calculated for each participant, and IOR magnitude was compared between age groups. Specifically, to calculate IOR effects, the mean response time at the cued location was obtained, and then compared to the average mean response time from the remaining 20 cue-target distances. A repeated measures ANOVA with cue location and age group indicated that there was a significant IOR effect, $F(1,63)=46.78, p<.001, \eta_{p}^{2}=.426$, where mean response time at the uncued location was faster than at the cued locations $\left(M_{\text {cued }}=457 \mathrm{~ms}\right.$, $\left.S D_{\text {cued }}=92 \mathrm{~ms}, M_{\text {uncued }}=439 \mathrm{~ms} ; S D_{\text {uncued }}=86 \mathrm{~ms}\right)$. The interaction between age group and cue location was non-significant, $F(1,63)=2.66, p=.108, \eta_{p}{ }^{2}=.040\left(M_{\text {cued-uncued older }}=22 \mathrm{~ms}\right.$, $S D_{\text {cued-uncued Older }}=23 \mathrm{~ms}, M_{\text {cued-uncued Younger }}=14 \mathrm{~ms} ; S D_{\text {cued-uncued Younger }}=20 \mathrm{~ms}$ ), suggesting the magnitude of the IOR effect was similar across both age groups.

\section{Age Differences in Attentional Slope}

To explore potential age differences in the spatial gradient of inhibition, the relationship between cue-target distance, and response time was analysed, and then compared between age groups. Figure 4 depicts mean response times at each cue-target distance for the two age groups. Two hierarchical regressions, quantifying the relationship between cue-target distance, and response time, controlling for eye movements were run for each participant. The first run checked for the presence of any univariate or multivariate outliers in the participants' data. This allowed us to remove any extreme response time data which may have unduly influenced results. Univariate outliers were defined as those with Z-scores exceeding $+\backslash$ - 3.29. Multivariate outliers were defined as those with Mahalanobis distance scores exceeding 5.99. These outliers were removed, and the second run produced the cue- 
THE SPATIAL SPREAD OF ATTENTION AND AGEING

target distance regression coefficients used in later analyses. The eye movement variable was entered into the first block to control for any eye movements made, and the cue-target distance variable into the second block. The distance coefficient represents the unique influence of cue-target distance on response times, indicating the slope of inhibition surrounding the cued location, and thus attentional spread.

It is important to recognise that the mean response time differed between the two age groups, and that overall response times and regression slopes in the IOR paradigm are typically related (Wilson et al., 2016). This difference in mean response time is likely due to generalised slowing with age (Verhaeghen \& Salthouse, 1997). Likewise, rather than reflecting age changes in attentional spread, it is possible that changes in visual system with age may have influenced the findings observed in the current experiment. ${ }^{1}$ While the majority of participants reported having normal or corrected to normal vision, five older adults reported issues with eye health, such as being diagnosed with macular degeneration, glaucoma, or having cataracts. Furthermore, across the lifespan, contrast sensitivity, as well as the functionality of visual pathways in the brain decline, particularly for fine detail processing (Elliott, Whitaker, \& MacVeigh, 1990; Elliott \& Werner, 2010; Owsley, 2011). Although the experiment was designed with the intention of controlling for these factors, such as increasing the contrast of the presented stimuli, as well as presenting the stimuli predominantly in para-foveal vision, it is possible that the eye health of some of the older adult participants unduly influenced the observed results.

Consequently, we compared age differences in inhibitory slope, while including mean response times and self-reported eye health issues as covariates. There was a significant influence of response time. $F(1,61)=4.26, p=.044, \eta_{p}^{2}=.065$, suggesting that age related slowing may account for some of the variance in the inhibitory slopes observed (Verhaeghen 
THE SPATIAL SPREAD OF ATTENTION AND AGEING

\& Salthouse, 1997). In contrast, the influence of eye health was non-significant, $F(1,61)=$ 0.003. $p=.953, \eta_{p}^{2}=0.00$. Finally, and crucially, when mean response time and eye health were controlled for the influence of age group on inhibitory slopes was significant, $F(1,61)=$ 7.25, $p=.009, \eta_{p}^{2}=.106,\left(M_{\text {older }}=-.122, S D_{\text {older }}=.087, M_{\text {younger }}=-.077, S D_{\text {younger }}=.094\right)$. This is strong evidence to suggest that when the appropriate controls are in place, older adults exhibited a strongly, significantly narrower spread of attention relative to older adults. exist.

We also compared inhibitory slopes when mean RT and eye health were not controlled for. An independent samples $t$-test was conducted to compare the relationship between age group (older vs. younger), and the value of the cue-target distance coefficient.. Overall, the difference in regression coefficients between the two groups was marginally significant, $t(63)=-1.97, p=.053, d=0.49$, where for older adults the slope value was greater. This provides converging evidence that, even when known age changes are not controlled for, older adults had a narrower spread of attention than younger adults.

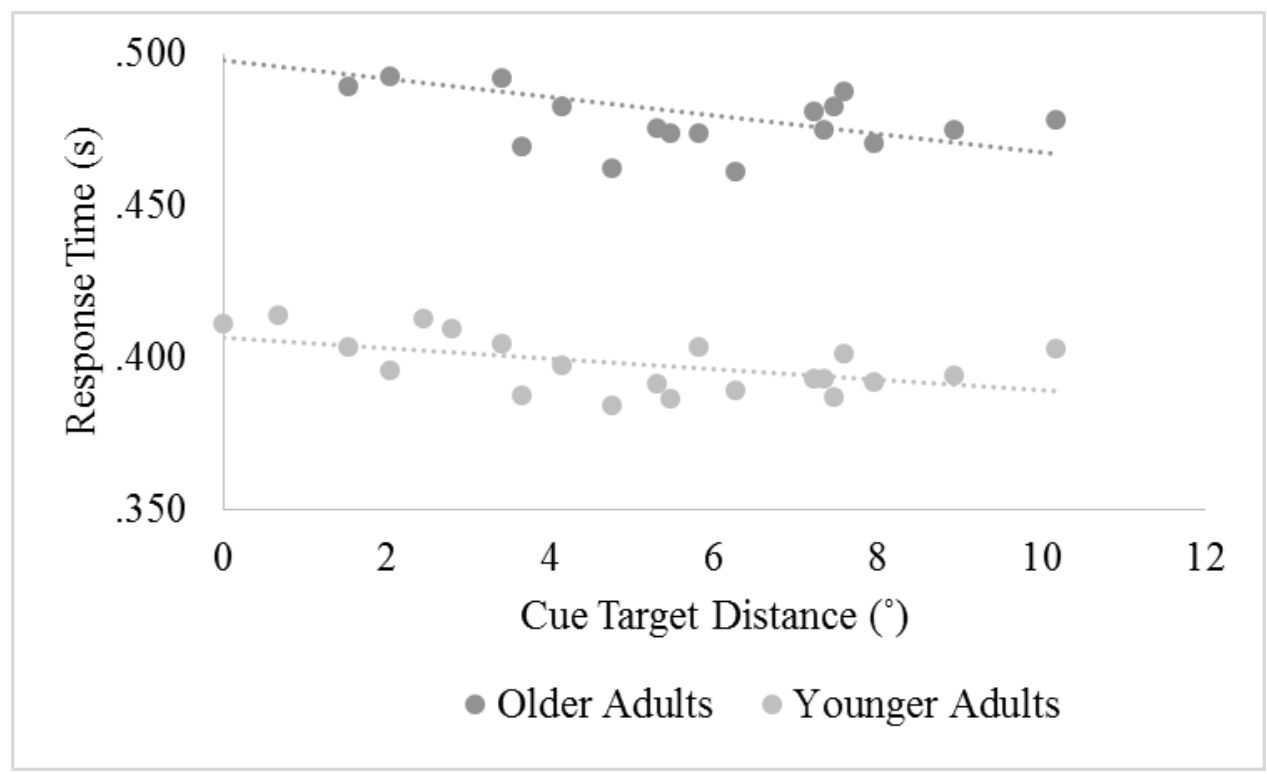

Figure 4. Experiment One: Mean response time as a function of cue-target distance for the younger and older adult groups. 
THE SPATIAL SPREAD OF ATTENTION AND AGEING

Interestingly, the obtained slopes in the current study differ in magnitude to that of previous work using this method (e.g. Taylor et al. 2015; Wilson et al. 2016). One reason for these differences in slope may have been the time difference between cue and target presentation in the current experiment compared to previous work. That is, while Wilson et al. (2016) utilised a cue-target delay of $800 \mathrm{~ms}$, the current experiment cue-target delay was 1315ms. Given that Taylor et al. (2015) demonstrated that the slope of inhibition differs across varying cue-target delays, where inhibitory slope becomes increasingly shallow with increased time delays, this would explain the relatively shallower slope obtained across both age groups.

Finally, to explore whether the control for eye movements influenced the pattern of results obtained, each participant's regression analysis was re-run without the eye movement covariate included. Here, the between subjects factor was age group, and the covariates included were mean response time, and eye health. Under this analysis, the effect of mean response time was non-significant, $\mathrm{F}(1,65)=1.73, \mathrm{p}=.193, \eta_{p}^{2}=0.028$, as was the effect of eye health, $\mathrm{F}(1,65)=0.03, \mathrm{p}=.872, \eta_{p}{ }^{2}=0.00$. However, the effect of age group on inhibitory slopes was marginally significant, $\mathrm{F}(1,65)=3.98, \mathrm{p}=.051, \eta_{p}^{2}=0.061$. This suggests that even when eye movements are not controlled for, there is an age difference in inhibitory slopes, and thus, attentional spread. However, when covariates were removed from the analysis, the age difference was minimal, $t(63)=-1.67, p=.100, d=0.42$. This is surprising given empirical work which suggests that the slope of inhibition does not differ for perceptual and motor IOR (Wang, Hilchey, Cao, \& Wang, 2014). Nonetheless, here we believe that by controlling for trials where eye movements were made, we were able to get a cleaner measure of covert attention. Thus, it may be that when covert attention is closely measured, there is a higher sensitivity to detect age differences in covert inhibitory spread. 
THE SPATIAL SPREAD OF ATTENTION AND AGEING

\section{Implicit Learning of Cue-Target Contingencies}

In the current paradigm, the cue was predictive of target location above chance level. Therefore, on each trial, it was more likely that the target fell in the previously cued location than any other location. This is important to note, as IOR effects are only typically seen when attention is exogenous (i.e. automatic, and not driven by top down goals; Posner \& Cohen, 1984). This probability manipulation may have had an overall effect on the IOR gradient for both groups, where the younger adult group better learnt the implicit probabilities inherent to the task over and above that of the older adult group. For instance, response times for target detection were actually faster, when the cue and target fell at the same location, compared to the other potential cue-target distances. This is turn, may have lead to shallower IOR slopes overall. Further, a recent review by Howard and Howard (2013), notes that while many processes of implicit learning are preserved with healthy ageing, other processes, for example, the learning of second order probabilities declines with healthy ageing. Nonetheless, in the current study, it unlikely that the probability manipulation drove age differences in attentional gradient. Specifically, if the younger adults were superior in implicitly learning cue-target probabilities, we would expect their attention to be narrowed in to the cued locations to a greater degree than the older adults. Consequently, this would cause them to display a relatively narrower distribution of attention. However, in contrast to these predictions, we obtained the opposite pattern of results, where older adults showed a narrower distribution of attention compared to younger adults. Therefore, given our obtained results, our data strongly suggests that older adults have a relatively restricted distribution of attention in comparison to younger adults. This conclusion is also supported by Experiment 2 (see below).

\section{Covert and Overt Attention}


THE SPATIAL SPREAD OF ATTENTION AND AGEING

Compared to previous studies of spatial IOR and ageing, in the current study, eye movements were measured via an analysis of central fixation patterns. Specifically, we asked participants to maintain fixation, while also measuring eye movements and then statistically controlling for any eye movements that were made. Given that there appears to be a difference in attentional and oculomotor inhibition of return (for example, Hunt \& Kingstone, 2003), it is possible that the current study was measuring a different form in IOR compared to that of previous work. However, given that recent research indicates that the spatial spread of inhibition shows similar patterns with, and without eye movements (Wang et al. 2014), we believe that this does not render our work and previous work incomparable. Instead, we believe that by controlling for eye movements, we can be more confident that we measured a form of inhibition that more accurately captures the deployment of covert spatial attention, with a higher degree of sensitivity compared to past research.

Furthermore, it is important to recognise recent research which has shown that when covert, perceptual IOR is measured, IOR effects have been found to be minimal when placeholders are removed from the visual display (e.g. Hilchey et al. 2016). This is in contrast to our study, which found substantial IOR effects without placeholder stimuli. One potential reason for this discrepancy is that the current study adopted a more sensitive measure of IOR compared to Hilchey et al. (2016). That is, while Hilchey and colleagues only measured changes in IOR magnitude across three locations, we tested across 40 possible locations, and 21 cue-target distances. Indeed, in Hilchey and colleagues' experiments, participant's attentional resources need only have been spread equally, to the bounds of the three locations, thus minimising potential for IOR magnitude effects to be found. In contrast, our study contained far more locations, making it more likely that subtle attentional differences may 
THE SPATIAL SPREAD OF ATTENTION AND AGEING

emerge. Thus, it appears that using a sensitive measure of attentional gradient across multiple cue-target locations may be able to tap into subtler changes in IOR than previous research.

\section{Summary}

Overall, the data from experiment one provides compelling evidence that older and younger adults distribute their attentional resources differently. While younger adults distributed their attention broadly to complete the IOR task, older adults focused their attention narrowly. This is in contrast to previous research that found minimal age differences in attentional spread. The key difference between Experiment 1 and past research was the absence of placeholders to cue attention. Specifically, the use of placeholders to cue attention had the potential to restrict the range over which attention was spread, thus lowering the sensitivity of the method to detect any group level differences. Here, for the first time, we have demonstrated that when placeholders are absent, inhibitory slope differs for younger and older adults, with older adults having steeper slopes. This implies that in comparison to younger adults, older adults show a relatively restricted spread of attention.

Nonetheless, while the presence versus absence of placeholders was the primary methodological difference between the current and previous studies, without a direct comparison of placeholder present versus placeholder absent experimental conditions, it is still possible that other factors are driving the observed age difference in Experiment 1. Given that there are sensory changes with healthy ageing, age differences in vision, rather than attention, may have driven the obtained findings. Specifically, there is typically a reduction in contrast sensitivity with healthy ageing (Owsley, 2011). This may have made stimuli not appear as bright for older compared to younger adults, leading them to have a narrower scope of attention. ${ }^{2}$ Therefore, Experiment Two sought to address this by comparing age 
THE SPATIAL SPREAD OF ATTENTION AND AGEING

differences in attentional slope for both placeholder present, and placeholder absent conditions. For the placeholder present condition, if sensory factors were driving the observed age differences, the stimuli would still appear dimmer to older adults, and thus lead older adults to have a narrower scope of attention. However, if no age difference are observed in the placeholder present condition, it is more likely that potential differences are driven by attentional processes. Thus, if, as we predict, placeholder presence restricts the spread of attention, age differences should only emerge when placeholders are absent from the visual display. In contrast, when placeholders are present, we expect age differences in attentional slope to disappear. This would provide strong converging evidence that placeholders are a key factor driving the distribution of attention, while explaining the previously obtained, null age differences in attentional slope.

\section{Experiment 2}

\section{Method}

\section{Participants}

Participants were from recruited from the general public and Australian National University, and were offered either course credit, or payment for participation. The ethical aspects of the research were approved by the Australian National University Human Research Ethics Committee. Participants included 30 younger adults, and 30 older adults. A summary of demographic data for the participant groups used in the main analysis is presented in Table

2.

Table 2

Demographic data of participants included in final analyses of Experiment 2 
Younger $\quad$ Older sig.

\begin{tabular}{|c|c|c|c|}
\hline Mean Age (Years) & $\mathrm{M}=21.90(\mathrm{SD}=3.56)$ & $\mathrm{M}=69.23(\mathrm{SD}=5.84)$ & - \\
\hline Total Years Education & $\mathrm{M}=16.85(\mathrm{SD}=3.12)$ & $\mathrm{M}=18.30(\mathrm{SD}=3.22)$ & .082 \\
\hline Ethnicity & Western $=12$ & Western $=28$ & - \\
\hline Gender & $\mathrm{F}=23, \mathrm{M}=6, \mathrm{O}=1$ & $\mathrm{~F}=24, \mathrm{M}=6$ & - \\
\hline GDS & $\mathrm{M}=3.5(\mathrm{SD}=3.35)$ & $\mathrm{M}=1.17 \mathrm{SD}=(1.66)$ & $.001 *$ \\
\hline MMSE & $M=28.67(S D=1.54)$ & $\mathrm{M}=28.97(\mathrm{SD}=0.72)$ & $.339 *$ \\
\hline Eye Health & $\mathrm{N}=0$ & $\mathrm{~N}=11$ & - \\
\hline Hearing Impairment & $\mathrm{N}=0$ & $\mathrm{~N}=7$ & - \\
\hline Cognitive Health & $\mathrm{N}=0$ & $\mathrm{~N}=0$ & - \\
\hline Psychological Illness & $\mathrm{N}=4$ & $N=2$ & - \\
\hline High Blood Pressure & $\mathrm{N}=0$ & $\mathrm{~N}=7$ & - \\
\hline Diabetes & $\mathrm{N}=1$ & $\mathrm{~N}=1$ & - \\
\hline Arthritis & $\mathrm{N}=0$ & $\mathrm{~N}=10$ & - \\
\hline
\end{tabular}

Notes. Ethnicity refers to whether participants identified with an Eastern or Western culture, or both. Eye health refers to participants who reported having cataracts, glaucoma, macular degeneration, or another uncorrected eye health condition. Cognitive health refers to participants who reported having Parkinson's disease, dementia, or mild cognitive impairment. To test between group differences, independent t-tests were conducted for 
THE SPATIAL SPREAD OF ATTENTION AND AGEING

continuous variables. Where necessary, these tests were corrected for violations of equal variance.

\section{Stimuli and Apparatus}

Stimuli were presented on an Apple Macintosh computer with an LCD monitor of dimensions $475 \mathrm{~mm}$ by $270 \mathrm{~mm}$, and refresh rate of $60 \mathrm{~Hz}$. Participants were seated comfortably, $600 \mathrm{~mm}$ from the monitor, with their head movements stabilised using a chin rest. Stimuli were presented using MATLAB and the psychophysics toolbox (Brainard \& Vision, 1997) set to a black background with a luminance of $0.36 \mathrm{~cd} / \mathrm{m}^{2}$. The method was largely similar to Experiment 1, however, there were some changes. Firstly, we did not include an eye tracking component. Secondly, fewer cue-target locations were sampled. This allowed us to measure a IOR slopes with a high degree of sensitvity, while lowering fatigue effects for participants. Stimuli were presented within a rectangular 5 x 5 grid, with dimensions $10^{\circ} \times 10^{\circ}$ of visual angle. The cues were presented at the centre of each quadrant of the grid while targets were presented at each intersection point of the grid. This led to a total of 100 possible cue-target combinations, and 10 possible cue target distances.

At the beginning of each trial, participants a grey, central fixation dot subtending a diameter of $0.10^{\circ}$, and a luminance of $77.41 \mathrm{~cd} / \mathrm{m}^{2}$ was presented for $500 \mathrm{~ms}$. In the placeholder absent condition, the cue, a green annulus with luminance $257.80 \mathrm{~cd} / \mathrm{m}^{2}$, a diameter of $0.48^{\circ}$, and thickness of $0.05^{\circ}$, was then presented for $100 \mathrm{~ms}$. Following this, there was a delay of $1317 \mathrm{~ms}$, following which the target, a white circle with luminance $358.98 \mathrm{~cd} / \mathrm{m}^{2}$, and diameter $0.48^{\circ}$ was presented until the participant responded, or $1000 \mathrm{~ms}$ had ellapsed. If participants made anticipatory responses (i.e. they reacted during the delay period), a warning message, reminding participants to wait for the white circle, appeared on 
THE SPATIAL SPREAD OF ATTENTION AND AGEING

the screen and remained for 3000ms. In the placeholder present condition, the same stimuli were used, except that now, at the beginning of each trial, placeholder stimuli appeared at each of the possible four cue locations, and remained on screen for the duration of the trial. The placeholder stimuli were green annuli, with diameter $0.48^{\circ}$ and thickness $0.048^{\circ}$, and a luminance of $30.73 \mathrm{~cd} / \mathrm{m}^{2}$.

\section{Procedure}

The experiment consisted of four blocks, two practice blocks corresponding to two experimental blocks. Placeholder presence and absence were blocked, and order of completion of the two blocks was counterbalanced. Firstly, participants completed a practice block consisting either of 10 placeholder present, or placeholder absent trials (corresponding to which condition they were about to complete). Participants then completed the first experimental block. This consisted of 240 trials containing either placeholders, or no placeholders. Of these, 200 trials were cue-target trials, while 40 trials were cue-only trials. For cue-target trials, it was equally likely for any possible cue target combination to occur. In comparison to Experiment 1, this miminised the chance that any age differences in cue-target contingency learning would influence results. Following this, participants completed the remaining practice block and experimental block. Within experimental blocks, self paced rest breaks were offered to participants every 60 trials completed. Overall, the experiment took approximately 1 hour to complete. 
THE SPATIAL SPREAD OF ATTENTION AND AGEING

\section{Results and Discussion}

\section{Accuracy Data}

First, accuracy data for the experiment were analysed ${ }^{3}$. Accuracy across both experimental conditions was high for both age groups. No single participant's accuracy fell below $90 \%$ in either the placeholder absent or placeholder present conditions, thus, no data were excluded from further analyses. A mixed ANOVA indicated no differences in accuracy across the placeholder present versus absent conditions, $F(1,58)=0.96, p=.332 ., \eta_{p}{ }^{2}=.016$, and a trend level interaction between placeholder and age group, $F(1,58)=3.22, p=.078$. $\eta_{p}{ }^{2}=.053$. Likewise, participants showed high accuracy for the target absent trials across both age groups and conditions, with no single participant's accuracy falling below 90\%. Therefore, no data were excluded from further analyses for this reason. A mixed ANOVA indicated no differences in trial absent accuracy across the placeholder present versus absent conditions, $F(1,58)=0.07, p=.790, \eta_{p}{ }^{2}=.001$, nor an interaction between placeholder and age group, $F(1,58)=.00, p=.703, \eta_{p}^{2}=.00$. Accuracy data for Experiment 2 are summarised in Table 3.

Table 3

Accuracy data for the placeholder present versus absent conditions in Experiment 2

Younger $\quad$ Older $\quad$ sig.

Placeholder Absent

$\mathrm{M}=99.17(\mathrm{SD}=.76) \quad \mathrm{M}=99.32(\mathrm{SD}=0.90)$

.487

Condition: Trial Present 
THE SPATIAL SPREAD OF ATTENTION AND AGEING

Placeholder Absent

$$
M=98.33(S D=1.83) \quad M=99.17(S D=1.78)
$$

Condition: Trial Absent

Placeholder Present

$$
\mathrm{M}=98.88(\mathrm{SD}=1.23)
$$$$
\mathrm{M}=99.40(\mathrm{SD}=0.94)
$$

Condition: Trial Present

Placeholder Present

$M=98.75(\mathrm{SD}=2.05) \quad \mathrm{M}=99.08(\mathrm{SD}=2.13)$

.539

Condition: Trial Absent

Notes. Independent samples t-tests were conducted to compare accuracy between age groups across the two placeholder conditions.

\section{Response Time Data}

Response time data was analysed to confirm an overall IOR effect for participants, and to explore whether any potential age differences existed in the magnitude of this IOR effect. Z-scores were calculated for response times across all cue-target distances. Any Zscores exceeding +/- 3.29 were excluded from further analysis. For the remaining data sets, overall mean reaction times were calculated. There was a main effect of placeholders on reaction time, $F(1,58)=8.37, p=.005, \eta_{p}^{2}=.126$, and age group, $F(1,58)=39.73, p<.001$, $\eta_{p}{ }^{2}=.406$. As shown in Table 4, overall, older adults had slower mean response times compared to younger adults. The interaction between placeholders and age group was nonsignificant, $F(1,58)=0.99, p=.323, \eta_{p}^{2}=.017$.

Table 4

Mean response times (ms) for placeholder absent and present conditions 


\section{Younger $\quad$ Older}

\begin{tabular}{lll}
\hline Placeholder Absent & $\mathrm{M}=354(\mathrm{SD}=38)$ & $\mathrm{M}=440(\mathrm{SD}=72)$ \\
Placeholder Present & $\mathrm{M}=363(\mathrm{SD}=47)$ & $\mathrm{M}=459(\mathrm{SD}=70)$
\end{tabular}

Next, IOR magnitude was compared between age groups for placeholder present and absent conditions. As shown in Figure 5, for the placeholder present condition, a mixed ANOVA revealed a main IOR effect, $F(1,58)=104.74, p<.001, \eta_{p}{ }^{2}=.644$, and a main effect of age group on response time, $F(1,58)=36.18, p<.001, \eta_{p}{ }^{2}=.384$. Critically, the interaction between IOR magnitude and age group was non-significant, $F(1,58)=1.74, p$ $=.193, \eta_{p}{ }^{2}=.029$, suggesting that the IOR effect was similar for both age groups in the presence of placeholders $\left(M_{\text {cued_older }}=511 \mathrm{~ms}, S D_{\text {cued_older }}=89 \mathrm{~ms}, M_{\text {uncued_older }}=459 \mathrm{~ms}\right.$, $S D_{\text {uncued_older }}=71 \mathrm{~ms}, M_{\text {cued_younger }}=403 \mathrm{~ms}, S D_{\text {cued_younger }}=56 \mathrm{~ms}$, Muncued_younger $=362 \mathrm{~ms}$, SDuncued_younger $=49 \mathrm{~ms})$. For the placeholder absent condition, a different pattern of results emerged, where there was a main effect of valid versus invalid trials, $F(1,58)=11.14, p=$. $001, \eta_{p}{ }^{2}=.161$, age group, $F(1,58)=37.09, p<.001, \eta_{p}{ }^{2}=.390$, and, crucially, an interaction between trial type and age group on response times, $F(1,58)=6.38, p=.014, \eta_{p}{ }^{2}=.099$. This suggests that the IOR effect differed between age groups when placeholders were absent. Follow-up paired samples $t$-tests indicated that while there was a significant IOR effect for the older adult group, $t(29)=3.40, p=.002, d=0.64 \quad\left(M_{\text {cued_older }}=462 \mathrm{~ms}, S D_{\text {cued_older }}=\right.$ 83ms, $\left.M_{\text {uncued_older }}=435 \mathrm{~ms}, S D_{\text {uncued_older }}=73 \mathrm{~ms}\right)$, this was not apparent for the younger adult group, $t(29)=0.804, p=.428, d=0.157\left(M_{\text {cued_younger }}=356 \mathrm{~ms}, \quad S D_{\text {cued_younger }}=46 \mathrm{~ms}\right.$, Muncued_younger $=352 \mathrm{~ms}$, sDuncued_younger $=38 \mathrm{~ms})$. 
THE SPATIAL SPREAD OF ATTENTION AND AGEING

Nonetheless, visual inspection of Figure 6 provides a possible explanation for this null finding. As can be seen, similar to Experiment 1, the younger adult group appeared to respond faster at the cued, compared to uncued locations. This is contradictory to what is expected in a typical IOR paradigm. While in Experiment 1 we speculated that this pattern of results may have resulted from unequal cue-target probabilities, resulting in implicit learning, this factor was ruled in out in Experiment 2. Thus, it is unclear why younger adults showed this pattern of results here. One possible explanation is due to the long-time delay between the cue and the target. While IOR effects are thought to be equivalent for younger and older adults at this cue-target delay, it is possible that here, younger adults' attention reoriented to the originally cued location following the long delay. In turn, this may have minimised the sensitivity of the pooled IOR magnitude measure to detect valid trial versus invalid trial effects. This is because the pooled IOR magnitude measure averaged over all of the possible non target locations which could lower the sensitivity of the measure to detect magnitude differences. Thus, to further explore IOR effects in Experiment 2, two secondary repeated measures analysis for the placeholder absent condition were run, which measured response times changes over all possible cue target distances for older and younger adult groups separately. In both analyses, sphericity assumption was violated, $\chi^{2}$ older $(44)=116.58, \mathrm{p}<$ $.001, \chi^{2}$ younger $(44)=68.17, p=.013$, and was corrected for using a Greenhouse-Geisser correction. The analyses revealed that both the younger and older adult groups experienced an IOR effect, $F_{\text {old }}(9,29)=9.57, p<.001, \eta_{p}{ }^{2}=.248, F_{\text {young }}(9,19)=4.67, p<.001 . \eta_{p}{ }^{2}=.139$. Altogether, this means that there was a significant IOR magnitude effect for both groups, whereby participants were generally slower around the cued closets cued locations, compared to the uncued locations further away. This is important as it confirms IOR occurred for both groups, subserving the main analysis where individual inhibitory slopes, reflecting attentional spread, are compared for the older and younger adults. 
THE SPATIAL SPREAD OF ATTENTION AND AGEING

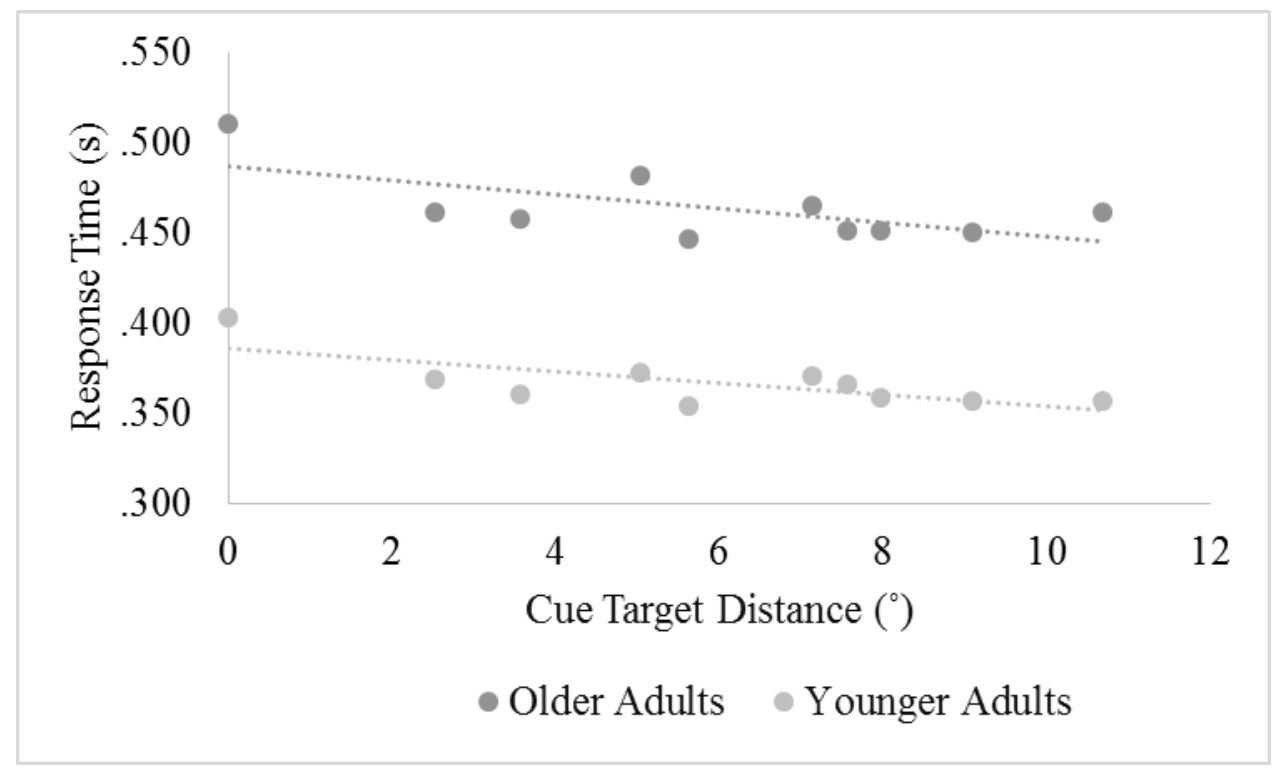

Figure 5. Experiment Two: Mean response time as a function of cue-target distance for the younger and older adult groups in the placeholder present condition.

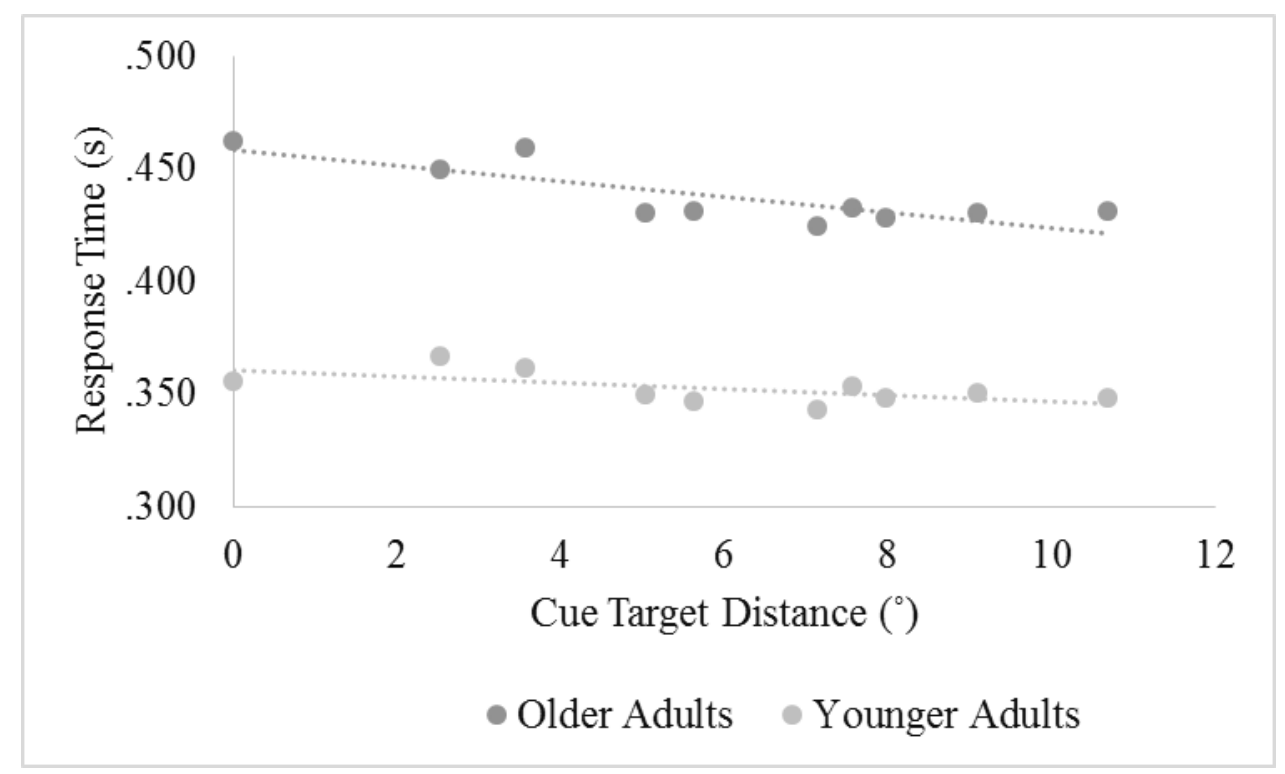

Figure 6. Experiment Two: Mean response time as a function of cue-target distance for the younger and older adult groups in the placeholder absent condition. 
THE SPATIAL SPREAD OF ATTENTION AND AGEING

\section{Age Differences in Attentional Slope}

To calculate individual participant inhibitory slopes for both the placeholder present and absent conditions, screened response time data were submitted to a linear regression, where cue-target distance was the independent variable, and response time was the dependent variable. A mixed ANOVA, with placeholders as a within subjects measure, and age group as a between subjects measure was then run to compare inhibitory slopes for the younger and older adults across the two experimental conditions. Overall, there was no main effect of placeholder presence versus absence, $F(1,58)=0.60, p=.443, \eta_{p}{ }^{2}=.010$, age group, $F(1,58)$ $=2.56, p=.138, \eta_{p}^{2}=.037$, or interaction between placeholders and age group, $F(1,58)=$ $2.50, p=.119, \eta_{p}{ }^{2}=.041$ on IOR slopes. However, given the relatively small sample size of the current study, it is possible that the reason for these null findings is a lack of statistical power. Furthermore, eye health, and mean response times in the placeholder absent and present conditions were not included as covariates here. This is because mean response times differed significantly as a function of both placeholder condition, making it inappropriate to calculate a combined response time measure to include in the analysis. Consequently, it is possible that the omnibus ANOVA, comparing slopes across placeholder conditions and age groups had a lowered sensitivity to detect potential age differences.

To address this, we conducted analyses separately for the placeholder present, and placeholder absent conditions, while controlling for age differences in mean response time and eye health. For the placeholder present condition, neither mean response time, $F(1,56)=$ $1.09, p=.302, \eta_{p}{ }^{2}=.019$, eye health, $F(1,56)=0.37, p=.570, \eta_{p}{ }^{2}=.006$, nor age group, $F(1$, $56)=0.59, p=.446, \eta_{p}{ }^{2}=.010$, significantly influenced inhibitory slopes $\left(M_{\text {older }}=-.09\right.$, $\left.S D_{\text {older }}=.07, M_{\text {younger }}=-.10 ; S D_{\text {younger }}=.07\right)$. This supports Langley et al. (2011), and Hartley 
THE SPATIAL SPREAD OF ATTENTION AND AGEING

and Kiely (1995), who found that when placeholders are present in the visual display, age differences in inhibitory slope are not observed.

Crucially, when placeholders were absent from the display, there was a main effect of age group on inhibitory slopes, $F(1,56)=5.60, p=.021, \eta_{p}{ }^{2}=.091$, where older adults had steeper slopes compared to younger adults $\left(M_{\text {older }}=-.13, S D_{\text {old }}=.10, M_{\text {younger }}=-.08 ; S D_{\text {younger }}\right.$ $=.08$ ). This provides strong, converging evidence with Experiment 1, that when placeholders are removed from the visual display, age differences in attentional spread emerge. The effects of mean response time, $F(1,56)=0.76, p=.388 . \eta_{p}^{2}=.013$, and eye health, $F(1,56)=0.87, p$ $=.356, \eta_{p}^{2}=.015$, were non-significant,

When eye health and mean response time were not controlled for, there were no age differences in inhibitory slopes for the placeholder present condition, $t(58)=0.104, p=.917$, $d=0.03$. Critically however, similar to Experiment 1 , in the placeholder absent condition, there was a marginally significant influence of age group on inhibitory slopes, $t(58)=-1.920$, $p=.060, d=0.50$, where older adults had a steeper slope compared to younger adults This indicates that when placeholders were absent from the visual display, older adults had a restricted attentional spread compared to younger adults. Thus, taken together, this is consistent with the results in Experiment 1, and provides converging evidence that placeholder presence versus absence influences the relationship between ageing and the slope of inhibition.

Finally, we combined the data from Experiment 1 and Experiment 2 placeholder absent conditions and reran our main analyses comparing inhibitory slopes for the younger and older participants groups ${ }^{4}$. To equate the experiments as much as possible, for Experiment 1, we used the inhibitory slopes which were calculated when eye movements 
THE SPATIAL SPREAD OF ATTENTION AND AGEING

were not controlled for. The model included age group and Experiment as between subject's effects, and mean response time and eye health as covariates. This allowed us to have higher statistical power to detect any potential age effects on attentional spread. Overall, this analysis revealed a main effect of age group on inhibitory slope, $F(1,119)=9.88, p=.002$, $\eta_{p}{ }^{2}=.077$. No other effects in the model were significant. As a whole, this provides convincing evidence that age group significantly influences inhibitory slopes, and thus, the spread of attention.

\section{General Discussion}

The aim of the current study was to better understand potential age differences in the spread of IOR, so to explore the relationship between healthy ageing and attentional distribution more generally. Previous studies have shown that the spatial spread of inhibition is largely preserved in healthy ageing. However, these studies employed placeholder stimuli to cue attention. This may have restricted attentional spread, and lowered the sensitivity of the paradigm to detect age differences. To address this, Experiment 1 compared age differences in inhibitory spread when placeholders were absent from the display. Experiment 2 then directly compared the influence of placeholder presence versus placeholder absence on inhibitory spread for both younger and older adults. Across both studies, our data indicates that when placeholders were absent from the visual display, inhibitory spread differs between younger and older adults, whereby attention is restricted for the older adult group. Further, Experiment 2 allowed us to control for age related sensory changes, thus allowing us to conclude that the observed age differences in the current data are likely to be driven by differences in attentional processing. 
THE SPATIAL SPREAD OF ATTENTION AND AGEING

That older and younger adults show differences in inhibitory spread is consistent with a range of studies using different methods to compare age differences in attentional distribution (Gottlob \& Madden, 1999; Greenwood \& Parasuraman, 1999, 2004; Hüttermann et al., 2012; Kosslyn et al., 1999; Pesce, et al., 2005). These studies typically require participants to process stimuli in disparate locations of the visual field simultaneously. For example, Hüttermann and colleagues recently developed a breadth of attention test to measure differences in older and younger adult populations. Following two instantaneous circular cues, participants were asked to identify the presence of target shapes in two spatially offset locations, equidistant from a central fixation cross. The maximum limit of attentional distribution was defined as the spatial distance between the two cued arrays where participants could identify the correct number of targets at an accuracy greater than $75 \%$. Nonetheless, by presenting targets at large spatial displacements in chorus, it was possible in this study, that rather than spreading attention, participants were shifting, or splitting their attentional resources between the two locations. Further, given that to measure the upper limits of attentional distribution, stimuli had to be presented at increasingly larger retinal eccentricities, it is not clear whether the observed age differences were a factor of visual declines, attention, or both. Given these uncertainties, the current study adds a unique piece of information to the literature, as it clearly demonstrates that neither peripheral stimuli, nor simultaneous location processing are necessary to observe age differences in attentional distribution.

The finding that younger adult versus the older adult group have different attentional distributions, where the younger adult resources encompass a broader range of the visual field, is likely to be based on declines in working memory with ageing (McDowd \& Shaw, 2000; Zanto \& Gazzaley, 2014). Indeed, Kreitz et al. (2015) have demonstrated a sizable 
THE SPATIAL SPREAD OF ATTENTION AND AGEING

positive correlation between performance in attentional spread tasks, and working memory tasks, where low working memory capacity predicted narrower attentional spread. Likewise, working memory capacity and attentional control have been related in a variety of tasks which indirectly involve a flexible allocation of attentional distribution (e.g Bleckley et al., 2003; Heitz \& Engle, 2007; Redick \& Engle, 2006; Rolle et al., 2017).

One recently-identified means of testing the relationship between working memory capacity and attention spread is to develop training programs that aim to improve working memory capacity, and subsequently, attentional flexibility (Rolle et al., 2017). That is, if working memory capacity improves with training, one would also expect to see a subsequent improvement on measures of attentional distribution. Indeed, by training both younger and older adults on a "distributed attention task”, Rolle et al. (2017) found marked improvement in both the capacity to focus and spread attention, as well as improvement in the performance of a spatial working memory task, after generalised slowing was accounted for. This strongly suggests that the processing of working memory and attention are tightly linked, and can explain the pattern of results observed in the current study. However, as we did not directly measure working memory capacity, nor did we measure and manipulate attention in a similar manner to Rolle et al. (2017), this conclusion can only be made tentatively. Future work exploring the relationship between working memory capacity, working memory load, and the distribution of inhibition in perceptual tasks is necessary to understand these potentially subtle relationships.

In the current study, a relatively broader distribution of attention would be most beneficial for efficient performance in the primary task (i.e. detecting the white circle). This is because the target could appear over a relatively large area of the central visual field, therefore, by distributing cognitive resources broadly, an individual would be more likely to 
THE SPATIAL SPREAD OF ATTENTION AND AGEING

detect the target on any given trial. Correspondingly, there is an obvious disadvantage to restricting the distribution of attention. This would require the participant to spend more time covertly scanning the visual field in order to detect a target. Therefore, the younger adult group’s broader spread of attention may reflect an efficient way to complete the task.

In comparison, the older adults use of a restricted attentional spread may have reflected an adaptation to physiological changes in the visual system. For instance, due to decreased peripheral acuity, older adults may have narrowed their attentional spread, thus experiencing attention akin to "tunnel vision" (Owsley, 2011). However, for the older adult population, there is a potentially positive role that a narrower distribution of attention would have: the relative increase in perceptual sensitivity that focused attention offers. Specifically, the Zoom Lens Model predicts that compared to a broad attentional spread, a narrow spread of attention will increase the visual systems capacity to resolve spatial detail (Eriksen \& James, 1986). Numerous behavioural and imaging studies support these claims (Balz \& Hock, 1997; Goodhew et al., 2017; Goodhew et al., 2016; Hock, Balz, \& Smollon, 1998; Müller et al., 2003).

Consistent with this notion of compensation, it is well known that the visual system declines with ageing, for both the physical optics of the eye and atrophy of occipital regions in the brain (Owsley, 2011). For example, older adults show decreased spatial contrast sensitivity with ageing, and more internal perceptual noise (Elliott, Whitaker, \& MacVeigh, 1990; Kline, Schieber, Abusamra, \& Coyne, 1983; Owsley, Sekuler, \& Siemsen, 1983; Sekuler \& Owsley, 1982; Tulunay-Keesey, Ver Hoeve, \& Terkla-McGrane, 1988, as cited in Owsley, 2011). If focused attention increases perceptual sensitivity, via processes such as contrast gain, it is therefore possible that a relatively narrow spread of attention may be more beneficial for the ageing brain, regardless of task, via a compensation of the declining visual 
THE SPATIAL SPREAD OF ATTENTION AND AGEING

system. This potential benefit of a smaller spread of attention is broadly consistent with compensatory models of ageing. Reuter-Lorenz and Cappell (2008) have developed a model of healthy ageing which posits that potential deficits of the ageing brain may be compensated for by the brain “working harder”, recruiting more neural resources to complete behavioural tasks to a satisfactory standard (Compensation-related utilization of neural circuits hypothesis, or CRUNCH). It is postulated that increases in neural activity would help to reduce the increased levels of internal perceptual noise that older adults encounter (ReuterLorenz \& Cappell, 2008). For instance, Grady et al. (1994) compared levels of neural activation for younger and older adults in perceptual processing of spatial locations and faces. They found older and younger adults to have equivalent behavioural performance, but differential neural activation, where in comparison to younger adults, older adults exhibited more activation of the frontal areas of the brain. Here, we suggest that a focused spread of attention may be related to such compensatory activity.

To conclude, our study provides strong evidence that ageing influences the capacity to distribute attentional resources in an inhibition of return task. This relationship holds, when both placeholders are removed from the visual display, and when eye movements are controlled for. Two potential reasons for these age differences may be differences in working memory capacity, and the resolving power of the visual system. Future work should more closely explore the link between working memory capacity and inhibitory spread, as well as the viability of training programs to enhance attentional flexibility. 
THE SPATIAL SPREAD OF ATTENTION AND AGEING 
THE SPATIAL SPREAD OF ATTENTION AND AGEING

\section{References}

Balz, G. W., \& Hock, H. S. (1997). The effect of attentional spread on spatial resolution. Vision Research, 37(11), 1499-1510. doi: 10.1016/S0042-6989(96)00296-9

Bennett, P. J., \& Pratt, J. (2001). The spatial distribution of inhibition of return. Psychological Science, 12(1), 76-80. doi: 10.1111/1467-9280.00313

Berlucchi, G. (2006). Inhibition of return: A phenomenon in search of a mechanism and a better name. Cognitive Neuropsychology, 23(7), 1065-1074. doi: $10.1080 / 02643290600588426$

Bleckley, M. K., Durso, F. T., Crutchfield, J. M., Engle, R. W., \& Khanna, M. M. (2003). Individual differences in working memory capacity predict visual attention allocation. Psychonomic Bulletin \& Review, 10(4), 884-889. doi: 10.3758/BF03196548

Brainard, D. H. (1997). The psychophysics toolbox. Spatial Vision, 10, 433-436. doi: $10.1163 / 156856897 x 00357$

Broadbent, D. E. (1982). Task combination and selective intake of information. Acta Psychologica, 50(3), 253-290.

Carrasco, M. (2011). Visual attention: The past 25 years. Vision research, 51(13), 1484-1525. doi: 10.1016/j.visres.2011.04.012

Castel, A. D., Chasteen, A. L., Scialfa, C. T., \& Pratt, J. (2003). Adult age differences in the time course of inhibition of return. The Journals of Gerontology Series B: 
THE SPATIAL SPREAD OF ATTENTION AND AGEING

Psychological Sciences and Social Sciences, 58(5), 256-259. doi: 10.1093/geronb/58.5.P256

Cave, K. R., \& Chen, Z. (2016). Identifying visual targets amongst interfering distractors: Sorting out the roles of perceptual load, dilution, and attentional zoom. Attention, Perception, \& Psychophysics, 78(7), 1822-1838. doi: 10.3758/s13414-016-1149-9

Cornelissen, F. W., Peters, E. M., \& Palmer, J. (2002). The Eyelink Toolbox: eye tracking with MATLAB and the Psychophysics Toolbox. Behavior Research Methods, 34(4), 613-617. doi: 10.3758\%2FBF03195489

Desimone, R., \& Duncan, J. (1995). Neural mechanisms of selective visual attention. Annual Review of Neuroscience, 18(1), 193-222. doi: 10.1146/annurev.ne.18.030195.001205

Downing, C. J. (1988). Expectancy and visual-spatial attention: effects on perceptual quality. Journal of Experimental Psychology: Human Perception and Performance, 14(2), 188-202. doi: 10.1037/0096-1523.14.2.188

Easterbrook, J. A. (1959). The effect of emotion on cue utilization and the organization of behavior. Psychological review, 66(3), 183-201. doi: 10.1037/h0047707

Egly, R., \& Homa, D. (1984). Sensitization of the visual field. Journal of Experimental Psychology: Human Perception and Performance, 10(6), 778-793. doi: 10.1037/0096-1523.10.6.778

Elliott, D., Whitaker, D., \& MacVeigh, D. (1990). Neural contribution to spatiotemporal contrast sensitivity decline in healthy ageing eyes. Vision Research, 30(4), 541-547. doi: 10.1016/0042-6989(90)90066-T 
THE SPATIAL SPREAD OF ATTENTION AND AGEING

Elliott, S. L., \& Werner, J. S. (2010). Age-related changes in contrast gain related to the M and P pathways. Journal of Vision, 10(4), 4-4. doi: 10.1167/10.4.4

Erel, H., \& Levy, D. A. (2016). Orienting of visual attention in aging. Neuroscience \& Biobehavioral Reviews, 69, 357-380. doi: 10.1016/j.neubiorev.2016.08.010

Eriksen, C. W., \& James, J. D. S. (1986). Visual attention within and around the field of focal attention: A zoom lens model. Perception \& Psychophysics, 40(4), 225-240. doi: 10.3758/BF03211502

Folstein, M. F., Folstein, S. E., \& McHugh, P. R. (1975). “Mini-mental state”: a practical method for grading the cognitive state of patients for the clinician. Journal of Psychiatric Research, 12(3), 189-198. doi: 10.1016/0022-3956(75)90026-6

Goodhew, S. C., Lawrence, R. K., \& Edwards, M. (2017). Testing the generality of the zoom-lens model: Evidence for visual-pathway specific effects of attended-region size on perception. Attention, Perception, \& Psychophysics, 79(4), 1147-1164. doi: 10.3758/s13414-017-1306-9

Goodhew, S. C., Shen, E., \& Edwards, M. (2016). Selective spatial enhancement: Attentional spotlight size impacts spatial but not temporal perception. Psychonomic Bulletin \& Review, 23(4), 1144-1149. doi: 10.3758/s13423-015-0904-6

Gottlob, L. R., \& Madden, D. J. (1999). Age differences in the strategic allocation of visual attention. The Journals of Gerontology Series B: Psychological Sciences and Social Sciences, 54(3), 165-172. doi: 10.1093/geronb/54B.3.P165 
THE SPATIAL SPREAD OF ATTENTION AND AGEING

Grady, C. L., Maisog, J. M., Horwitz, B., Ungerleider, L. G., Mentis, M. J., Salerno, J. A., . . . Haxby, J. V. (1994). Age-related changes in cortical blood flow activation during visual processing of faces and location. Journal of Neuroscience, 14(3), 1450-1462.

Greenwood, P., \& Parasuraman, R. (1999). Scale of attentional focus in visual search. Attention, Perception, \& Psychophysics, 61(5), 837-859. doi: 10.3758/BF03206901

Greenwood, P., \& Parasuraman, R. (2004). The scaling of spatial attention in visual search and its modification in healthy aging. Attention, Perception, \& Psychophysics, 66(1), 3-22. doi: 10.3758/BF03194857

Hartley, A. A., Kieley, J., \& Mckenzie, C. R. (1992). Allocation of visual attention in younger and older adults. Attention, Perception, \& Psychophysics, 52(2), 175-185. doi: $0.3758 \% 2 F B F 03206771$

Hartley, A. A., \& Kieley, J. M. (1995). Adult age differences in the inhibition of return of visual attention. Psychology and Aging, 10(4), 670-683. doi: 10.1037/0882-7974.10.4.670

Hedden, T., \& Gabrieli, J. D. (2004). Insights into the ageing mind: a view from cognitive neuroscience. Nature Reviews. Neuroscience, 5(2), 87-96. doi: 10.1038/nrn1323

Heitz, R. P., \& Engle, R. W. (2007). Focusing the spotlight: individual differences in visual attention control. Journal of Experimental Psychology: General, 136(2), 217-240. doi: 10.1037/00963445.136.2.217

Hilchey, M. D., Pratt, J., \& Christie, J. (2016). Placeholders dissociate two forms of inhibition of return. The Quarterly Journal of Experimental Psychology, 1-13. doi: 10.1080/17470218.2016.1247898 
THE SPATIAL SPREAD OF ATTENTION AND AGEING

Hock, H. S., Balz, G. W., \& Smollon, W. (1998). Attentional control of spatial scale: Effects on selforganized motion patterns. Vision Research, 38(23), 3743-3758. doi: 10.1016/S00426989(98)00023-6

Hoyer, W. J., Cerella, J., \& Buchler, N. G. (2011). A search-by-clusters model of visual search: Fits to data from younger and older adults. The Journals of Gerontology Series B: Psychological Sciences and Social Sciences, 66(4), 402-410. doi: 10.1093/geronb/gbr022

Hunt, A. R., \& Kingstone, A. (2003). Inhibition of return: Dissociating attentional and oculomotor components. Journal of Experimental Psychology: Human Perception and Performance, 29(5), 1068-1074. doi: 10.1037/0096-1523.29.5.1068

Huntsinger, J. R. (2013). Does emotion directly tune the scope of attention? Current Directions in Psychological Science, 22(4), 265-270. doi: 10.1177/0963721413480364

Hüttermann, S., Bock, O., \& Memmert, D. (2012). The breadth of attention in old age. Ageing Research, 3(1),67-70. doi: 10.4081/ar.2012.e10

Hüttermann, S., \& Memmert, D. (2015). The influence of motivational and mood states on visual attention: A quantification of systematic differences and casual changes in subjects' focus of attention. Cognition and Emotion, 29(3), 471-483. doi:10.1080/02699931.2014.92076

Jefferies, L. N., \& Di Lollo, V. (2015). When can spatial attention be deployed in the form of an annulus? Attention, Perception, \& Psychophysics, 77(2), 413-422. doi: 10.3758/s13414-0140790-4. 
THE SPATIAL SPREAD OF ATTENTION AND AGEING

Jefferies, L. N., \& Di Lollo, V. (2017). Deployment of spatial attention to a structural framework: exogenous (alerting) and endogenous (goal-directed) factors. Attention, Perception, \& Psychophysics. doi:10.3758/s13414-017-1378-6

Jefferies, L. N., Roggeveen, A. B., Enns, J. T., Bennett, P. J., Sekuler, A. B., \& Di Lollo, V. (2015). On the time course of attentional focusing in older adults. Psychological Research, 79(1), 2841. doi: 10.1007/s00426-013-0528-2

Kastner, S., \& Pinsk, M. A. (2004). Visual attention as a multilevel selection process. Cognitive, Affective, \& Behavioral Neuroscience, 4(4), 483-500. doi: 10.3758/CABN.4.4.483

Klein, R. M., Christie, J., \& Morris, E. P. (2005). Vector averaging of inhibition of return. Psychonomic Bulletin \& Review, 12(2), 295-300. doi: 10.3758/BF03196375

Kline, D. W., Schieber, F., Abusamra, L. C., \& Coyne, A. C. (1983). Age, the eye, and the visual channels: contrast sensitivity and response speed. Journal of Gerontology, 38(2), 211-216.

Kosslyn, S. M., Brown, H. D., \& Dror, I. E. (1999). Aging and the scope of visual attention. Gerontology, 45(2), 102-109.

Kreitz, C., Furley, P., Memmert, D., \& Simons, D. J. (2015). Working-memory performance is related to spatial breadth of attention. Psychological Research, 79(6), 1034-1041. doi: 10.1007/s00426-014-0633-x

LaBerge, D. (1983). Spatial extent of attention to letters and words. Journal of Experimental Psychology: Human Perception and Performance, 9(3), 371-379. doi: 10.1037/00961523.9.3.371 
THE SPATIAL SPREAD OF ATTENTION AND AGEING

LaBerge, D., \& Brown, V. (1989). Theory of attentional operations in shape identification. Psychological Review, 96(1), 101-124. doi: 10.1037/0033-295X.96.1.101

Langley, L. K., Gayzur, N. D., Saville, A. L., Morlock, S. L., \& Bagne, A. G. (2011). Spatial distribution of attentional inhibition is not altered in healthy aging. Attention, Perception, \& Psychophysics, 73(3), 766-783. doi: 10.3758/s13414-010-0059-5

Madden, D. J., \& Gottlob, L. R. (1997). Adult age differences in strategic and dynamic components of focusing visual attention. Aging, Neuropsychology, and Cognition, 4(3), 185-210. doi: $10.1080 / 13825589708256647$

Mattay, V. S., Fera, F., Tessitore, A., Hariri, A. R., Berman, K. F., Das, S., . . . Weinberger, D. R. (2006). Neurophysiological correlates of age-related changes in working memory capacity. Neuroscience Letters, 392(1), 32-37. doi: 10.1016/j.neulet.2005.09.025

McAuliffe, J., Chasteen, A. L., \& Pratt, J. (2006). Object-and location-based inhibition of return in younger and older adults. Psychology and Aging, 21(2), 406-410. doi: 10.1037/08827974.21.2.406

McCalley, L., Bouwhuis, D., \& Juola, J. F. (1995). Age changes in the distribution of visual attention. The Journals of Gerontology Series B: Psychological Sciences and Social Sciences, 50(6), 316-331. doi: 10.1093/geronb/50B.6.P316

McDowd, J. M., \& Shaw, R. J. (2000). Attention and aging: A functional perspective. In F. I. M. Craik \& T. A. Salthouse (Eds.), The handbook of aging and cognition (pp. 221-292). Mahwah, NJ: Lawrence Erlbaum Associates. 
THE SPATIAL SPREAD OF ATTENTION AND AGEING

Müller, N. G., Bartelt, O. A., Donner, T. H., Villringer, A., \& Brandt, S. A. (2003). A physiological correlate of the "zoom lens" of visual attention. Journal of Neuroscience, 23(9), 3561-3565.

Nicol, J. R., Watter, S., Gray, K., \& Shore, D. I. (2009). Object-based perception mediates the effect of exogenous attention on temporal resolution. Visual Cognition, 17(4), 555-573. doi: $10.1080 / 13506280802113860$

Owsley, C. (2011). Aging and vision. Vision Research, 51(13), 1610-1622. doi: 10.1016/j.visres.2010.10.020

Owsley, C., Sekuler, R., \& Siemsen, D. (1983). Contrast sensitivity throughout adulthood. Vision Research, 23(7), 689-699. doi: 10.1016/0042-6989(83)90210-9

Pesce, C., Guidetti, L., Baldari, C., Tessitore, A., \& Capranica, L. (2005). Effects of aging on visual attentional focusing. Gerontology, 51(4), 266-276. doi: 10.1159/000085123

Posner, M. I. (1980). Orienting of attention. Quarterly Journal of Experimental Psychology, 32(1), 325. doi: 1080/00335558008248231

Posner, M. I., \& Cohen, Y. (1984). Components of visual orienting. Attention and Performance X: Control of Language Processes, 32, 531-556.

Posner, M. I., Rafal, R. D., Choate, L. S., \& Vaughan, J. (1985). Inhibition of return: Neural basis and function. Cognitive Neuropsychology, 2(3), 211-228. doi: 10.1080/02643298508252866

Posner, M. I., Snyder, C. R., \& Davidson, B. J. (1980). Attention and the detection of signals. Journal of Experimental Psychology: General, 109(2), 160-174. doi: 10.1037/00963445.109.2.160 
THE SPATIAL SPREAD OF ATTENTION AND AGEING

Pringle, H. L., Irwin, D. E., Kramer, A. F., \& Atchley, P. (2001). The role of attentional breadth in perceptual change detection. Psychonomic Bulletin \& Review, 8(1), 89-95. doi: 10.3758/BF03196143

Quigley, C., Andersen, S. K., \& Müller, M. M. (2012). Keeping focused: sustained spatial selective visual attention is maintained in healthy old age. Brain Research, 1469, 24-34. doi: 10.1016/j.brainres.2012.06.019

Redick, T. S., \& Engle, R. W. (2006). Working memory capacity and attention network test performance. Applied Cognitive Psychology, 20(5), 713-721. doi: 10.1002/acp.1224

Reuter-Lorenz, P. A., \& Cappell, K. A. (2008). Neurocognitive aging and the compensation hypothesis. Current Directions in Psychological Science, 17(3), 177-182. doi: 10.1111/j.1467-8721.2008.00570.x

Rolle, C. E., Anguera, J. A., Skinner, S. N., Voytek, B., \& Gazzaley, A. (2017). Enhancing Spatial Attention and Working Memory in Younger and Older Adults. Journal of Cognitive Neuroscience, 29(9), 1483-1497. doi: 10.1162/jocn_a_01159

Sekuler, R., \& Owsley, C. (1982). The spatial vision of older humans. Aging and Human Visual Function, 185-202.

SR research Ltd. (2005-2008). EyeLink User Manual (version 1.4.0).

Taylor, J. E. T., Chan, D., Bennett, P. J., \& Pratt, J. (2015). Attentional cartography: mapping the distribution of attention across time and space. Attention, Perception, \& Psychophysics, 77(7), 2240-2246. doi: 10.3758/s13414-015-0943-0 
THE SPATIAL SPREAD OF ATTENTION AND AGEING

Theeuwes, J., Kramer, A. F., \& Belopolsky, A. V. (2004). Attentional set interacts with perceptual load in visual search. Psychonomic Bulletin \& Review, 11(4), 697-702. doi: 10.3758\%2FBF03196622

Tulunay-Keesey, U., Ver Hoeve, J. N., \& Terkla-McGrane, C. (1988). Threshold and suprathreshold spatiotemporal response throughout adulthood. JOSA A, 5(12), 2191-2200. doi: 10.1364/JOSAA.5.002191

Vanlessen, N., De Raedt, R., Koster, E. H., \& Pourtois, G. (2016). Happy heart, smiling eyes: a systematic review of positive mood effects on broadening of visuospatial attention. Neuroscience \& Biobehavioral Reviews, 68, 816-837. doi: 10.1016/j.neubiorev.2016.07.001

Verhaeghen, P., \& Salthouse, T. A. (1997). Meta-analyses of age-cognition relations in adulthood: Estimates of linear and nonlinear age effects and structural models. Psychological Bulletin, 122(3), 231-249. doi: 10.1037/0033-2909.122.3.231

Wang, B., Hilchey, M. D., Cao, X., \& Wang, Z. (2014). The spatial distribution of inhibition of return revisited: No difference found between manual and saccadic responses. Neuroscience Letters, 578, 128-132. doi: 10.1016/j.neulet.2014.06.050

Wilson, K. E., Lowe, M. X., Ruppel, J., Pratt, J., \& Ferber, S. (2016). The scope of no return: Openness predicts the spatial distribution of Inhibition of Return. Attention, Perception, \& Psychophysics, 78(1), 209-217. doi: 10.3758/s13414-015-0991-5

Yesavage, J. A., \& Sheikh, J. I. (1986). 9/Geriatric depression scale (GDS) recent evidence and development of a shorter version. Clinical Gerontologist, 5(1-2), 165-173. doi: 10.1300/J018v05n01_09?journalCode=wcli20 
THE SPATIAL SPREAD OF ATTENTION AND AGEING

Zanto, T. P., \& Gazzaley, A. (2014). Attention and ageing. The Oxford Handbook of Attention, 927971 
THE SPATIAL SPREAD OF ATTENTION AND AGEING

\section{Acknowledgements}

This research is supported by an Australian Government Research Training Program Scholarship awarded to R.K.L This research was supported by an Australian Research Council (ARC) Discovery Early Career Researcher Award (DE140101734), and a Future Fellowship (FT170100021) awarded to S.C.G. The authors would like to thank Dr. Kim Keily for his advice and guidance. 


\section{Notes}

1. We thank an anonymous reviewer for these insights

2. We thank an anonymous reviewer for this suggestion

3. Two participants were erroneously run on only $50 \%$ of placeholder absent trials. However, given that the participants still completed 120 trials, we included their data in analyses. Further, one participant completed a slightly modified version of the experiment. For this participant, in both the placeholder present and absent conditions, two of the possible 100 cue-target combinations were not completed. These trials were replaced with a random selection of the remaining 98 cue-target combinations.

4. When adding the data from Experiment 1 and Experiment 2 for combined analyses, we note that a small number of participants completed both Experiment 1 and Experiment 2. 